\title{
From university to company: education of optical communications in cooperation with industry at Technical University of Ostrava
}

Vladimir Vasinek, Jan Skapa, Petr Siska, Frantisek Hanacek, Jan Latal, et al.

Vladimir Vasinek, Jan Skapa, Petr Siska, Frantisek Hanacek, Jan Latal, Petr Koudelka, Iva Petrikova, "From university to company: education of optical communications in cooperation with industry at Technical University of Ostrava," Proc. SPIE 9666, 11th Education and Training in Optics and Photonics Conference, 96660Y (5 June 2009); doi: 10.1117/12.2208030 


\title{
From university to company - education of optical communications in cooperation with industry at Technical University of Ostrava
}

\author{
Vladimir Vasinek, Jan Skapa, Petr Siska, Frantisek Hanacek, Jan Latal, Petr Koudelka,Iva \\ Petrikova, Department of Telecommunications, Technical University of Ostrava, Czech \\ Republic
}

\begin{abstract}
Paper deals with cooperation between companies and university, especially with interactions companies and students, companies and pedagogues. At present it is possible to observe insufficient level of practical skills and knowledge among students and their pedagogues, there is no articulation for companies' demands. We try to solve this situation with the help of pilot compartment. Its main task is to associate university teachers, graduate students and companies'specialists. Within the scope activities of the compartment is to prepare one or two day's long special courses. Their mass point is focused to practical training; prepare conditions for trainee-ships dedicated to teachers and students on one side and special courses for technicians, dealers and companies' management on the other. The main goal of this compartment is an interconnection between university education and requirements out coming from praxis. There are many ways of how to fulfill such cooperation.
\end{abstract}

Keywords: university education, interconnection between university and companies, practical teaching, laboratory education

\section{INTRODUCTION}

Department of Telecommunications cooperates with many telecommunication companies for many years. This cooperation is based on applied research or innovation development only. For education purposes it has only a small meaning, main goal is to obtain knowledge about solved problematic and to obtain money for further development of department. For now our department is relatively well equipped with many devices and apparatus, especially in fiber optics and optical communications. Except of fiber optic hardware (laser sources, LED sources, optical powermeters, OTDR-meters, fusion-splices, Raman OTDR-meter, optical tables, benches, etc.) our department is working with SW for designing of optical routes, systems, fibers and devices. We are using Optiwave SW as a multilicence for a group of 10 students.

Teachers in our department prepare many study texts, syllabi and laboratory instruments. But they have a small contact with requirements of praxis. According to our experiences the situation is the same for all universities in Czech Republic. Our department is better equipped with devices in comparison to small and middle companies and may be compared with large telcos. The problem is that educational background is not used for creation of cooperation in education for practice and vice versa. This lack of cooperation has been the main motor for project of new type of cooperation between university department and companies.

\section{DESCRIPTION OF PROJECT}

As it has been mentioned above nowadays we may observe quite missing system and systematic cooperation with praxis as a basis for education in telecommunications and optical communications. The cooperation is 
based on occasional personal bonds between individual teachers and companies. The consequences of such state are limited or zero knowledge of university teachers about requirements of firms. The problem is more serious because of teachers read their lectures according to their best effort. We are trying to change this situation and to achieve two partial goals of the same importance.

a. To increase practical knowledge in optical communications for students and their teachers

b. On the basis of outstanding higher skill level of teachers to reach the state when department will be natural expert authority for optical communications sought out the firms in treatment problems with improving of skills for their staff

Both partial goals may be subdivided into individual goals.

Ad a.

- Creation of study materials for practical teaching both in classical and e-learning form

- To integrate important experts from firms and companies to opposition procedures of these educational materials

- To create natural space for student's involvement to managed practice in firms and future employers

- To create space for future short-terms stages of teachers in firms and companies

Ad b.

Creation of specialized study materials for short terms educational activities (one or two days typically) according to firm's requirements oriented to practical training and evaluate their efficiency in educational process

- Creation of organizing scheme, structure and background for further education of university teachers and researchers

\section{$2.1 \quad$ Content of courses}

All courses are prepared on the base of existing lectures. The main ideas of such conception are following:

Introduction to telecommunications and optical fibers - Telecommunications, basic ideas, connection point to point, networks, information capacity of optical fibers, transition from analog to digital transmission systems, place and role of the optical communication systems, history and development of the transmission properties and parameters of optical fibers, fiber optical communications systems, basic block diagram of optical transmission, nowaday stage in optical systems, future development trends

Digest of light and its properties - Plane electromagnetic wave, refractive index, energy transmission by light wave, linearly polarized light, circular and elliptical polarized light, transmission of light through quarterwave plate, interference of light, two-beam interference, coherence of light, Gauss beam, Gauss beam difraction, light as beam of rays, refractive index, reflection and diffraction of light, Brewster angle, total internal reflection, slight as a stream of photons, energy levels of electron and transitions among them, absorption and emission of light

Basics of optical fibers 1 - Light guidance in optical fibers, step index fibers as a basic optical communication structure, total reflection on the core-cladding boundary, refractive index of core and cladding, critical angle on the core-cladding boundary and critical angle of transmission, light launching into optical fiber, acceptance angle, numerical aperture, attenuation on optical fibers, bending losses, microbending losses, light scattering, basic mechanism of absorption, transparent windows for fibers made of $\mathrm{SiO} 2$, calculation of total attenuation of optical line, direct method of attenuation measurement

Basics of optical fibers 2 - Intermodal and chromatic dispersion, description of modes, number of modes, physical meaning of modes, modal dispersion, mechanismus of pulse spreading and its calculations, resctriction of the bite rate due to modal dispersion in optical line, first solution to modal dispersion - gradeindex fibers, basic ideas of graded-index fibers, reduction of the modal dispersion due to graded-index fibers, second and principal solution of the modal dispersion - singlemode fibers, structire of singlemode fiber, comparison of the modal dispersion in three types of optical fibers

Basics of optical fibers 3 - Chromatic dispersion, mechanisms of chromatic dispersion creation, calculating pulse spreading caused by chromatic dispersion, definition of bit rate and bandwidth, relationship between bit 
rate and bandwidth, electrical and optical bandwidth, total dispersion and bit rate, bit rate calculation, specification of bit rate and bandwidth by optical fibers producers

Basics of optical fibers 4 - Reading a data sheet of optical fibers, general parameters, optical characteristics, geometrical characteristics of optical fibers, environmental specifications of optical fibers, mechanical specifications, other characteristics

Advanced description of optical fibers 1 - Maxwell's equations, interpretation of Maxwell's equations, wave equations, solving wave equations, plane waves, propagation of the EM waves, wave equations for timeharmonic EM field, distribution of electrical and magnetical part of wave in space, wave propagation in lossy medium, phase and group velocity of waves, group refraction index, EM waves in bounded media, rectangular waveguide, modes in waveguide, cutoff frequency and waveguide wavelengths, advanced description of total internal reflection, effects on boundary of two dielectrics, phase shift in total reflection, evanescent waves, reflectances and transmittances on boundary of two dielectrics, Goos-Haenchen shift

Advanced description of optical fibers 2 - Cylindrical fibers, modes of cylindrical fibers, LP modes, selecting rules for EM waves in optical fibers, waves and rays of optical fibers, meridional and skew rays, guided, radiation and leaky modes, optical power in fiber, power distribution between core and cladding, number of modes and measuremnt of attenuation

Advanced description of optical fibers 3 - Cutoff wavelength and cutoff normalised frquency of optical fiber, cutoff condition and total internal reflection, power confinement and cutoff condition, role of cladding, effective refractive index

Advanced description of optical fibers 4 - Attenuation in multimode fibers, general approach to attenuation, intrinsic losses, Rayleigh scattering, behavior of $\mathrm{SiO} 2$ fibers, choice of operating wavelength, other materials for optical fibers production, fluoride fibers, chalkogen fibers, extrinsic absorption, macrobending loss, microbending loss, influence of mode structure to fiber attenuation, attenuation and attenuation constant, dispersion in multimode fibers, total dispersion and pulse width, electrical and optical bandwidth, mechanism of modal dispersion

Advanced description of optical fibers 5 - Chromatic dispersion, derivation of the formula for material dispersion, practical calculation of material dispersion according to datasheets, Sellmeier relations, influence of source spectral width, waveguide dispersion, description of bit rate and bandwidth in multimode fibers, choice of operating wavelength according to fiber dispersion, dispersion power penalty

Basics of singlemode fibers (SM fibers) - Principle of action, Gaussian beam, influence of core and cladding to mode field diameter (MFD), cutoff wavelength of SM fiber, attenuation in SM fibers, macrobending loss, microbending loss, absorption of light and light scattering of SM fibers, dispersion and bandwidth, chromatic dispersion, material dispersion, waveguide dispersion, conventional fibers, shifted fibers, dispersion flattened fibers, polarization mode dispersion (PMD), bandwidth and bit rate of SM fibers, reading of datasheets, general characteristics, transmission parameters, MFD and cutoff wavelength, geometric characteristics, mechanical properties of SM fibers

Advanced description of SM fibers 1 - Mode field, Gaussian model and real mode field distribution, cutoff wavelength and V-number, effective cutoff wavelength, detailed description of SM fiber attenuation, bending losses for step-index SM fiber, more sophisticated refractive index profiles of SM fibers, coping with dispersion in Sm fibers, dispersion solution for WDM fibers

Advanced description of SM fibers 2 - compensation of chromatic dispersion with compensating fibers (DCF), designing of DCF systems, dispersion compensating gratings (DCG), fiber Bragg gratings (FBG), production of FGB fibers, polarization mode dispersion (PMD), PMD characteristics of SM fiber, polarizationmaintaining fibers, PMD compensation, nonlinear effects in SM fibers, nonlinear refractive effects, self phase modulation (SPM), solitons in optical fiber, cross-phase modulation (XPM), four-wave mixing (FWM) in SM fiber, stimulated scattering effects, trends in fiber design

Fabrication of optical fibers and cables 1 - Requirements on technologies, preparation of molten glasses, fusible technologies, fiber drawing, drawing of long fibers - double-crucible method, deposition technologies, OVPO, VAD, MCVD, PCVD technologies, optical fibers coatings, primary and secondary tight coating, optical cables for telecommunications and data transmission, basic structure of optical cable

Fabrication of optical fibers and cables 2 - Mechanical parameters of optical cables, protection and humidity resistivity, gels in optical cables, protection of optical cables to biological influences, materials for cable coatings, PVC, polyurethan (PUR), HDPE, nylon, teflon, LSZH materials, examples of optical cable datasheets 
Installation methods and procedures with optical cables, Installation methods, installation in climbing-irons, cable laying into protective pipes, protection to lightning, self-supporting cables, cables for chemical industry, cables for higher temperature, blowing technologies, new cable laying technologies of optical cables, structure of telecommunication optical lines, structured cabling systems, optical switchgears, design of optical lines, attenuation budget, total dispersion budget

Optical fiber cable connectorization 1 - Mounting splices, fixed splices, intrinsic and extrinsic losses in splices, reflection loss, insertion loss, optomechanical splices, fused splices, fusion splicers of optical fibers, fusion splice protection, optical connectors, basic structure of optical connectors, insertion loss of optical connector, return loss

Optical fiber cable connectorization 2 - Distribution curve of insertion loss, repeatability of connection, mounting density of optical connectors and their compatibility with optical cables and appliances, types and materials for optical ferrules, types of optical connectors-SMA, FC, ST, SC, FDDI, ESCON, E2000, new types of optical connectors-MTRJ, MTP, MU, MINIMAC, the latest trends in connections with optical connectors

Tests and measurements of optical fibers and cables- 1 Kinds of measurements, measurement at SM and MM optical cables, attenuation measurement, conditions for correct attenuation measurement, cut off method, direct method of attenuation measurement, OTDR-metr, basic parts of OTDR-metr, dead zones

Tests and measurements of optical fibers and cables -2 Curve routing of backscattering, cable factor, shape of faults on the backscattering curve, chromatic dispersion, methods for chromatic dispersion measurement, differential phase shift, time delay of pulses, BERT, measurement of insertion loss of connectors and other passive elements in optical lines, return loss measurement

Basics of sources for optical communications - lasers Basic properties of lasers, spontaneous and stimulated radiation, population inversion, positive feedback, material loss, laser activity and conversion laser characteristics, characteristics and laser light properties, diode lasers, basic structures of laser diodes, $\mathrm{DH}$, $\mathrm{BH}$, stripe lasers, QW lasers, VCSEL, optical resonators, restriction of the bandwidth, DFB and DBR lasers, laser diodes with and without active cooler, datasheets parameters of laser diodes

Basics of sources for optical communications -LEDs Advantages and disadvantages of LEDs to laser dodes, creation of photons in semiconductors, light radiation from pn junction, basic description and LED characteristics, homostructure LEDs, heterostructure LEDs, surface radiating LEDs(SLED), edge radiating LEDs(ELED), light coupling into optical fiber, technology improving coupling of optical fiber to LEDs, datasheets parameters of LEDs, modulating bandwidth

Sources for optical communications-advanced description 1 - Intrinsic semiconductors: Fermi energy levels and number of charge carriers, charge carriers density, effective mass of electrons and holes, doped semiconductors, materials for optical communications, PN junction unbiased, density of electrons and holes, biased PN junction, direct and indirect transitions, conservation of energy and momentum law, E-k diagram, Einstein's rate relations, two-levels systems, equilibrium radiation from two-levels system, relationship between spontaneous and stimulated radiation, emission and absorption of radiation, absorption coefficient and linear coefficient of material amplification

Sources for optical communications-advanced description 2 - Conditions for light amplification, population inversion-description, population inversion and methods of its realisation, three levels and four levels systems, optical feedback, phenomena in optical resonator, threshold condition for laser amplification, losses in optical resonator, total amplification and population inversion, amplification coefficient for small signals

Sources for optical communications-advanced description 3 - Efficiency of laser diodes-internal, external, differential, power, laser resonator efficiency, threshold amplification and line width, radiating and nonradiating recombinations and their influence to laser diodes efficiency, conditions for high laser efficiency, heterosctuctures and their function, efficiency in terms of an E-k diagram, characteristics of laser diodes, threshold current and operating current of laser diode, spectral properties of LDs, radiation patterns of LD, longitudinal and transverse modes of laser radiation, LD modulation

Sources for optical communications-advanced description - 4 Theoretical limit of laser modulation rate, analog and digital modulation of $L D s$, rate equations of $L D$, relaxation oscillation of $L D$, Transfer function of $L D$, intensity modulation of $L D$, chirp, noise of $L D, R I N$ and its description, transmitter modules, function block diagram, driving circuits of LD transmitters, link codes for data transmission, circuit solution of optical transmitters with LEDs and LDs 
Sources for optical communications-advanced description - 5 Modulation circuits, controlling and monitoring circuits, coupling light from LED transmitter, coupling light from laser transmitter, back reflection protection of LD, integral characteristic of optical transmitter-eye diagram, faults of optical transmitters and their consequences in eye-diagram, internal and external modulators, Mach-Zehnder modulator, electro absorption modulator

Basics of detectors and receivers for optical communications - Basic requirements on optical detectors, basic principles and fotodetector structures, photodiode with PN junction, photovoltaic and photoconductive modes of operation, responsivity of photodiode, responsivity versus wavelength, short and long cutoff wavelength of photodiode, sensitivity of photodiode, photodiode bandwidth, equivalent circuit of a PN photodiode, bandwidth and photodiode digest, PIN photodiode, avalanche photodiode APD), MSM detectors Detectors and receivers for optical communications - advanced description 1 SNR a error rate of receiver, photodiode noise sources, shot noise, thermal noise, dark current, noise $1 / \mathrm{f}$, noise equivalent circuit, total noise figure, normalized noise value, SNR for PIN photodiode, SNR for APD, dependence of SNR on APD amplification, noise equivalent power (NEP), bit error rate (BER), BER and decision threshold, error function (erf), complementary error function (erfc)

Detectors and receivers for optical communications - advanced description 2 - BER description - Q parameter, relationship between BER and SNR, BER versus Q parameter, minimum optical power - photodiode sensitivity, minimum number of photons per bit, quantum limit

Receiver units for optical communications - Functional block diagram of optical receiver, input receiver unit, equivalent circuits of an optical front end, quantizer, clock recovery, decision circuit design, datasheets and parameters of optical receivers

Components of fiber optic networks 1 Point to point links, fiber optical networks, TDM and WDM, add/drop problems, repeaters and amplifiers in optical networks and signal amplifiers, passive and active elements of fiber optical networks, transceivers for fiber optic networks, transmitter requirements in WDM networks, tunable lasers, receivers for optical networks, receiver requirements in WDM networks, amplifiers in optical networks, functional types of optical amplifiers, semiconductor optical amplifiers(SOA), SOA advantages and disadvantages

Components of fiber optic networks 2 Fabry-Perot amplifier(FPA), travelling-wave amplifier(TWA), Gain of FPA, gain of TWA, gain saturation, FPA bandwidth, TWA bandwidth, crosstalk, polarization dependent gain, noise in optical amplifiers, amplified spontaneous emission ASE, datasheets and parameters of optical amplifiers

Components of fiber optic networks 3 Erbium doped fiber amplifiers (EDFA), principle of operation, energy band structure, amplifier pumping, C-band and L-band, gain and noise in EDFAs, EDFA components, splicing of erbium-doped fibers, ways to reduce splicing loss, pump laser diodes, datasheets and parameters of EDFAs, other types of optical networks amplifiers

Passive components of fiber optic networks 1 Fused biconical tapers and couplers, port configurations of couplers, characteristics of a WDM coupler, datasheets parameters of couplers, basics of WDM coupler theory, coupling length, phase mismatch, WDM multiplexers and demultiplexers, broadband narrowband WDM MUX/DEMUX

Passive components of fiber optic networks 2 Mux/Dmux, WDM waveguide gratings, WDM diffraction gratings, Fiber Bragg grating (FBG), WDM MUX/DEMUX applications, Add/Drop MUX/DEMUX and routers, optical filters, thin-film filters, tunable filters, acoustooptical filters, optical isolators, optical circulator, attenuators, variable attenuator, datasheet parameters, optical switches and functional modules, wavelength converters

Fiber optic networks Telephone networks, data networks, access networks, transport networks, multiplexing hierarchy in telecommunication networks, metropolitan networks, synchronous and asynchronous network, modulation and optical network multiplex, cable TV networks, place of optical networks in OSI model, coherent communication systems

Fiberless optical networks Atmosphere as a transmission media, phenomena limiting range and bit rate, directional and diffused networks, sources and detectors for fiberless networks, principles of design for fiberless networks 


\section{WHY COOPERATION BETWEEN DEPARTMENT AND COMPANIES}

As it has been mentioned above, nowadays is no managed connection between department and companies in educational process. Study materials are not consulted or disputed by firms even if these materials will be used for laboratory education or other practical training. The consequence is that the disaffection of university education from firm's requirements is increasing. That manifests both by students and their teachers.

Firms and companies (as consumers of products of university educational systems) instead of appealing to suitable university departments solve better their questions and problems of staff further education by their selves. Authors of this paper have been opponents of many educational technical projects for companies and met with such effect dominantly. In any project figured university as a educational institution in spite of it acts about technical education.

This state may be improved by managed process only. This process provides educational materials according to requirements of practice. It is necessary to work with three task groups - the first one is created by students, the second one by their teachers and the third one by technicians.

\section{Students}

They will be integrated within direct educational process. The main idea is to increase the laboratory and experimental part of their study including project teaching, managed stages, bachelor and diploma work are coming out from needs of firms and companies. Students' motivation is the same as motivation to study university; authors of this paper have experiences that the student motivation is higher if study is more practically oriented.

Teachers

At present many teachers have never been outside university and knew no needs of firms and companies and ways of thinking of technicians outside universities. Any forced compulsion of teachers to absolve stages in firms and companies have no meaning if it not created natural place for meeting of both sides. As the starting point it is necessary to put the new prepared study materials for rout of technicians not of other teachers. It is impossible to continue with state when study materials especially pro practical teaching are disputed by other teachers. Further it is necessary for teachers to be students for several days. The goal is creation the short time activities for technicians at higher educational level. Motivation for teachers is a real obtaining of contacts, knowledge and skills about ways of thinking.

Technicians

Their main motivation is possibility to influence the study process as their future employers, possibility to create system for further education of their own staff and possibility to create real setting for creation of expert teams. Part of this group will be addressed with offer to participate in educational activities for the first groups, the rest will be participated as a regular students in short term courses.

\section{CONCLUSION}

This project is a pilot project at the Technical University of Ostrava. If the project is successful it will be spread to other departments. For Department of telecommunications in case of success it will be created new source of real cooperation with firms and companies. The starting point will be improvement in educational process; other points are creation expert teams and solving difficult science and technical tasks. 